\title{
Einstein Oscillators in Thallium Filled Antimony Skutterudites
}

\author{
Raphaël P. Hermann, ${ }^{1}$ Rongying Jin, ${ }^{2}$ Werner Schweika, ${ }^{3}$ Fernande Grandjean, ${ }^{1, *}$ David Mandrus, ${ }^{2}$ \\ Brian C. Sales, ${ }^{2}$ and Gary J. Long ${ }^{4}$ \\ ${ }^{1}$ Department of Physics, B5, University of Liège, B-4000 Sart-Tilman, Belgium \\ ${ }^{2}$ Solid State Division, Oak Ridge National Laboratory, Oak Ridge, Tennessee 37831 \\ ${ }^{3}$ Institut für Festkörperforschung, Forschungszentrum Jülich $\mathrm{GmbH}$, D-52425 Jülich, Germany \\ ${ }^{4}$ Department of Chemistry, University of Missouri-Rolla, Rolla, Missouri 65409-0010
}

(Received 20 December 2002; published 3 April 2003)

\begin{abstract}
Inelastic neutron scattering measurements on several $\mathrm{Tl}$ filled skutterudites $\left(\mathrm{Tl}_{0.5} \mathrm{Co}_{3.5} \mathrm{Fe}_{0.5} \mathrm{Sb}_{12}\right.$, $\mathrm{Tl}_{0.8} \mathrm{Co}_{3} \mathrm{FeSb}_{12}$, and $\mathrm{Tl}_{0.8} \mathrm{Co}_{4} \mathrm{Sb}_{11} \mathrm{Sn}$ ) all show a sharp peak in the vibrational density of states at $57 \pm$ $2 \mathrm{~K}$, which is absent in the unfilled skutterudite $\mathrm{CoSb}_{3}$. Heat capacity measurements on $\mathrm{Tl}_{0.8} \mathrm{Co}_{4} \mathrm{Sb}_{11} \mathrm{Sn}$ as compared to $\mathrm{CoSb}_{3}$ are consistent with the presence of a localized vibrational mode associated with the "rattling" thallium atoms in this filled skutterudite compound. Both results are well described by a localized Einstein mode model with an Einstein temperature $\Theta_{E}$ of $53 \pm 1 \mathrm{~K}$. These data provide perhaps the clearest example of local mode behavior in a concentrated metallic system.
\end{abstract}

DOI: 10.1103/PhysRevLett.90.135505

PACS numbers: 63.20.Pw, 61.12.-q, 65.40.Ba

The concept of a phonon glass and electron crystal [1] is instrumental in designing highly efficient thermoelectric materials, materials that must exhibit a small thermal conductivity and a large electrical conductivity. The $R M_{4} \mathrm{Sb}_{12}$ filled antimony skutterudite compounds, where $R$ is a rare earth or an electropositive element such as thallium and $M$ is a transition metal, offer a crystallographic structure, which fulfills the requirements for a phonon glass [2]. Indeed, the unit cell contains 34 atoms and the rare earth [3] or thallium [4] atom is weakly bound in an oversized cage formed by $12 \mathrm{Sb}$ atoms. The presence of this filling "rattler" atom causes a dramatic reduction in the lattice contribution to the thermal conductivity without significantly affecting the semiconducting properties of the unfilled $\mathrm{CoSb}_{3}$ skutterudite. It is believed that incoherent localized vibrations associated with the filling atom are responsible for the reduction in the thermal conductivity.

Inelastic neutron scattering and heat capacity measurements are two of the very few techniques capable of investigating the existence of these localized vibrational modes. They were used [3] to study the localized vibrational modes of lanthanum in $\mathrm{LaFe}_{4} \mathrm{Sb}_{12}$ through a comparison with $\mathrm{CeFe}_{4} \mathrm{Sb}_{12}$ for the inelastic neutron scattering, and with $\mathrm{CoSb}_{3}$ for the heat capacity measurements. Unfortunately, these two compounds differ in the nature either of the rare earth or the transition metal.

Herein, we present both inelastic neutron scattering and heat capacity measurements on $\mathrm{CoSb}_{3}$ and $\mathrm{Tl}_{0.8} \mathrm{Co}_{4} \mathrm{Sb}_{11} \mathrm{Sn}$, which, apart from the $\mathrm{Tl}$ filling atom, differ only by at most one tin atom out of 12 antimony atoms within the skutterudite lattice, a tin atom which is known [5] to be located on the antimony sublattice. Further, we present inelastic neutron scattering measurements on $\mathrm{Tl}_{0.8} \mathrm{Co}_{3} \mathrm{FeSb}_{12}$ and $\mathrm{Tl}_{0.5} \mathrm{Co}_{3.5} \mathrm{Fe}_{0.5} \mathrm{Sb}_{12}$, mea- surements that help to unambiguously assign the peak observed in the inelastic neutron scattering results to the rattling thallium atom. Thallium containing skutterudites were chosen for this work because the thallium scattering cross section is $\sim 95 \%$ coherent which means that any scattering angle independent features in the inelastic scattering spectrum must be related to incoherent motion of the thallium atoms. The samples used in this study are the same as those synthesized and characterized earlier [4].

The heat capacity measurements were carried out between 2 and $100 \mathrm{~K}$ with the heat capacity option of the Physical Property Measurement System from Quantum Design system. According to the specifications, the relative error on the heat capacity measurements on this instrument is less than $2 \%$. The thallium contribution to the heat capacity has been determined by measuring the difference in the heat capacities of $\mathrm{Tl}_{0.8} \mathrm{Co}_{4} \mathrm{Sb}_{11} \mathrm{Sn}$ and $\mathrm{CoSb}_{3}$ (see Fig. 1). Below $20 \mathrm{~K}$, see the inset of Fig. 1, the difference significantly exceeds the error bars and is successfully modeled by the Einstein law,

$$
C_{E}(T)=A x^{2} e^{x} /\left(e^{x}-1\right)^{2},
$$

with an amplitude $A$ of $0.8 \times 3 R=19.9 \mathrm{~J} / \mathrm{mol} \mathrm{K}, x=$ $\Theta_{E} / T$, and $\Theta_{E}=53(1) \mathrm{K}$, where $\Theta_{E}$ is the Einstein temperature. At temperatures well above $20 \mathrm{~K}$, the Einstein contribution is only $0.8 / 16.8$ or $4.8 \%$ of the total heat capacity of $\mathrm{Tl}_{0.8} \mathrm{Co}_{4} \mathrm{Sb}_{11} \mathrm{Sn}$. This small contribution explains the increasing noise in the data of Fig. 1 at higher temperatures. When we take into account the heat capacity at higher temperatures and vary $A$, a fit with Eq. (1) yields values of $\Theta_{E}$ and $A$ which depend on the fitting range. The resulting average values are $\Theta_{E}=56 \pm$ $2 \mathrm{~K}$, and $A=22 \pm 1 \mathrm{~J} / \mathrm{mol} \mathrm{K}$, values which correspond to a slightly higher $\mathrm{Tl}$ filling of $0.88 \pm 0.04$. 


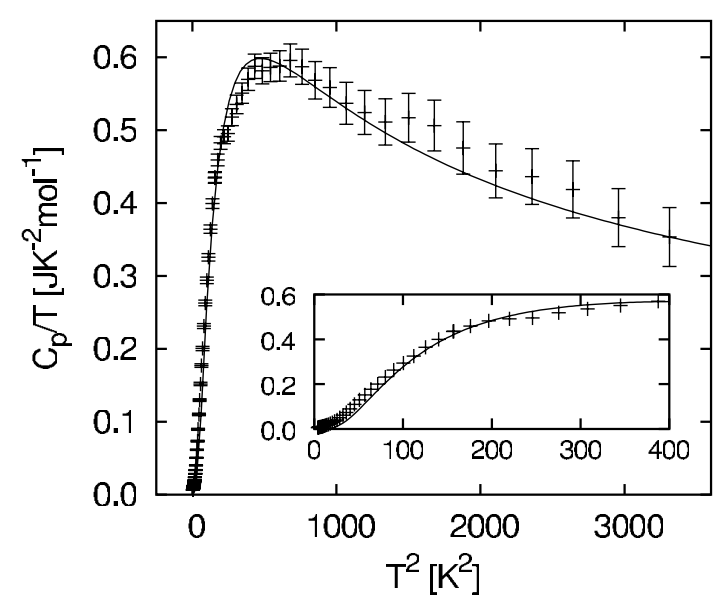

FIG. 1. The heat capacity difference between $\mathrm{Tl}_{0.8} \mathrm{Co}_{4} \mathrm{Sb}_{11} \mathrm{Sn}$ and $\mathrm{CoSb}_{3}$ divided by the temperature as a function of the square of the temperature. The solid line is the result of a fit with the Einstein model. Inset: An expanded view of the low temperature region.

The inelastic neutron scattering measurements were performed at the DIDO research reactor at Jülich, Germany. Only $1.5,1.8,1.5$, and $1.7 \mathrm{~g}$ of polycrystalline sample were available for $\mathrm{CoSb}_{3}, \mathrm{Tl}_{0.5} \mathrm{Co}_{3.5} \mathrm{Fe}_{0.5} \mathrm{Sb}_{12}$, $\mathrm{Tl}_{0.8} \mathrm{Co}_{3} \mathrm{FeSb}_{12}$, and $\mathrm{Tl}_{0.8} \mathrm{Co}_{4} \mathrm{Sb}_{11} \mathrm{Sn}$, respectively. The measurements were performed on the cold neutron time-of-flight instrument DNS [6]. At the chosen wavelength of $4.6 \AA$, the usable energy range is limited from -3.8 to $\sim 25 \mathrm{meV}$ in energy gained by the neutrons. The background and each sample were measured for $\sim 15 \mathrm{~h}$ at a temperature of $\sim 305 \mathrm{~K}$.

In order to extract the density of states, the inelastic neutron scattering experimental data were treated as follows. First, the scattering data was integrated over a scattering angle $2 \theta$ range of $30^{\circ}$ to $130^{\circ}$. Second, the amount of background to be subtracted was determined by balancing the density of states for neutrons with energy loss with that for neutrons with energy gain. Third, as the sample mass in the beam and the sample cross section were different for each sample, the four data sets were normalized so that the observed parabolic dependence of the density of states would be the same for each sample. The density of states, DOS, obtained for all four compounds over the 1 to $10 \mathrm{meV}$ energy range has been fitted with the function

$$
\operatorname{DOS}(\omega)=a \omega^{2}+\frac{2 b}{w \sqrt{\pi / \ln 2}} \exp \left[-4\left(\frac{E-\hbar \omega}{w}\right)^{2} \ln 2\right]
$$

which is the sum of the Debye dependence of the density of states in $\omega^{2}$ and a Gaussian of full width at half maximum, $w$, associated with an instrumentally broadened Einstein oscillator. As a result of the normalization, the arbitrary factor $a$ has the same value for all four compounds. The other parameters obtained from the fits are given in Table I.

The DOS, obtained from the inelastic neutron scattering measurements on $\mathrm{CoSb}_{3}, \quad \mathrm{Tl}_{0.5} \mathrm{Co}_{3.5} \mathrm{Fe}_{0.5} \mathrm{Sb}_{12}$, $\mathrm{TlCo}_{3} \mathrm{FeSb}_{12}$, and $\mathrm{TlCo}_{4} \mathrm{Sb}_{11} \mathrm{Sn}$, are shown in Fig. 2 as a function of the energy gained by the neutron during the scattering process. The insets of Fig. 2 show the Gaussian peak remaining after subtracting the Debye parabola, a peak which corresponds to the thallium localized vibrational modes. It should be noted that the observation of the local modes in Fig. 2 is independent of the chosen range of integration over the different scattering vectors.

The difference observed at $\sim 5 \mathrm{meV}$ between the three thallium filled compounds and $\mathrm{CoSb}_{3}$ is significant. Above about $10 \mathrm{meV}$, the Debye model for the DOS no longer applies. No significant difference between filled and unfilled compounds can be clearly identified above $10 \mathrm{meV}$, but the density of states for $\mathrm{Tl}_{0.8} \mathrm{Co}_{3} \mathrm{FeSb}_{12}$ and $\mathrm{Tl}_{0.8} \mathrm{Co}_{4} \mathrm{Sb}_{11} \mathrm{Sn}$ is about $10 \%$ smaller than for the unfilled skutterudite, $\mathrm{CoSb}_{3}$. Furthermore, the rattling mode in $\mathrm{Tl}_{0.8} \mathrm{Co}_{3} \mathrm{FeSb}_{12}$ is much sharper than in $\mathrm{Tl}_{0.8} \mathrm{Co}_{4} \mathrm{Sb}_{11} \mathrm{Sn}$ as indicated by the observed full widths at half maximum, $w$, given in Table I. The different width of the Tl localized vibrational mode results because, in the first case a Co atom is replaced by an $\mathrm{Fe}$ atom in the second neighbor shell, whereas, in the second case an Sb atom is replaced by a $\mathrm{Sn}$ atom in the first neighbor shell of the Tl. The instrumental resolution at an energy transfer of $5 \mathrm{meV}$ is $1.9 \mathrm{meV}$. By assuming a convolution of the instrumental and natural Gaussian linewidths,

$$
w_{\mathrm{obs}}^{2}=w_{\mathrm{ins}}^{2}+w_{\mathrm{nat}}^{2}
$$

TABLE I. The energy, in meV, and the corresponding temperature, in $\mathrm{K}$, of the thallium localized vibrational mode, the area, and the full width at half maximum of the associated Gaussian peak.

\begin{tabular}{lllll}
\hline \hline Compound & $E(\mathrm{meV})$ & $T(\mathrm{~K})$ & $b$ (arb. units) & $w(\mathrm{meV})$ \\
\hline $\mathrm{Tl}_{0.5} \mathrm{Co}_{3.5} \mathrm{Fe}_{0.5} \mathrm{Sb}_{12}$ & $5.0 \pm 0.1$ & $58 \pm 1$ & $0.20 \pm 0.02$ & $1.9^{\mathrm{a}}$ \\
$\mathrm{Tl}_{0.8} \mathrm{FeCo}_{3} \mathrm{Sb}_{12}$ & $4.9 \pm 0.1$ & $57 \pm 1$ & $0.26 \pm 0.02$ & $1.9 \pm 0.2$ \\
$\mathrm{Tl}_{0.8} \mathrm{Co}_{4} \mathrm{Sb}_{11} \mathrm{Sn}$ & $4.7 \pm 0.1$ & $55 \pm 1$ & $0.38 \pm 0.02$ & $3.0 \pm 0.2$ \\
\hline \hline
\end{tabular}

${ }^{\mathrm{a}}$ This value was constrained to the value found for $\mathrm{Tl}_{0.8} \mathrm{FeCo}_{3} \mathrm{Sb}_{12}$. 


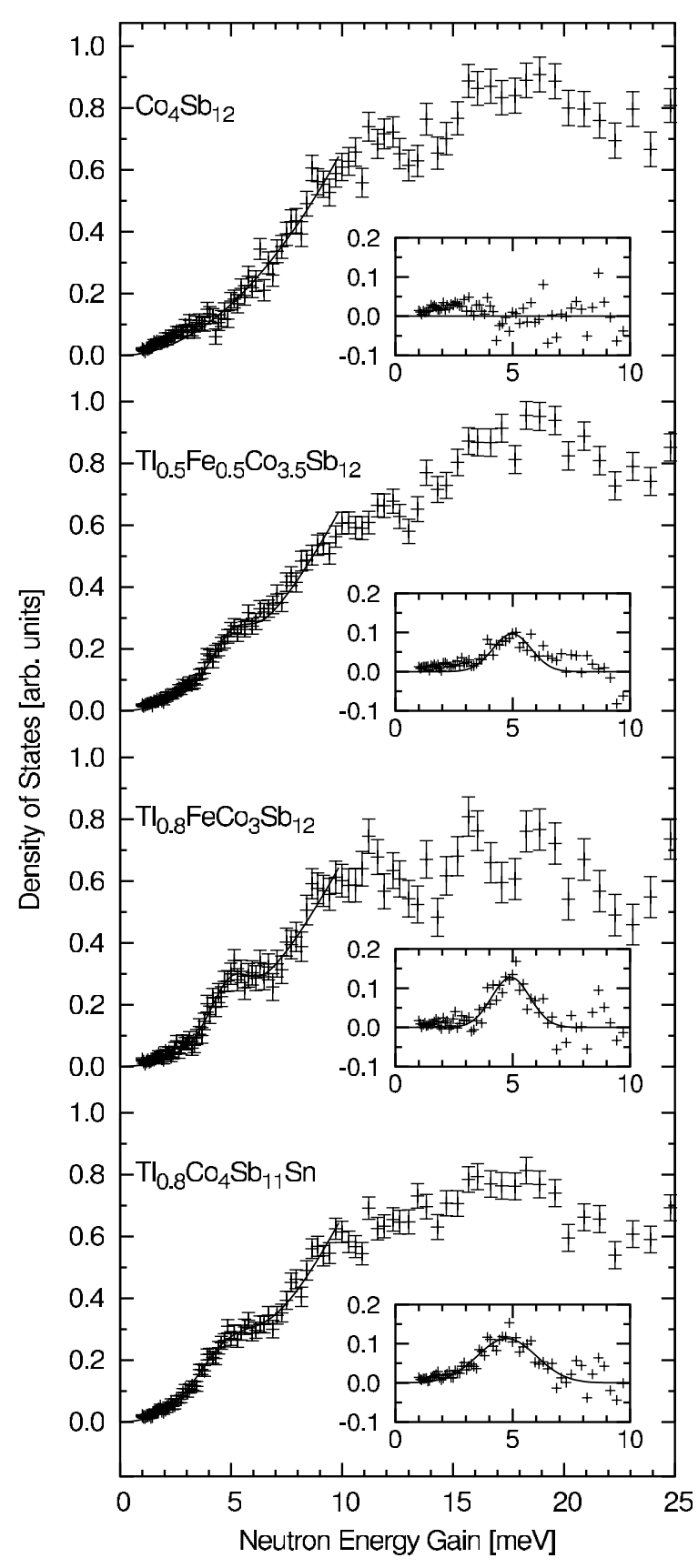

FIG. 2. The phonon density of states as obtained from the inelastic neutron scattering in $\mathrm{CoSb}_{3}, \mathrm{Tl}_{0.5} \mathrm{Co}_{3.5} \mathrm{Fe}_{0.5} \mathrm{Sb}_{12}$, $\mathrm{TlCo}_{3} \mathrm{FeSb}_{12}$, and $\mathrm{Tl}_{0.8} \mathrm{Co}_{4} \mathrm{Sb}_{11} \mathrm{Sn}$. The solid line is the result of a fit with Eq. (2). Insets: The Gaussian peak in the three thallium filled skutterudites.

the natural width, $w_{\text {nat }}$, of the mode in $\mathrm{Tl}_{0.8} \mathrm{Co}_{4} \mathrm{Sb}_{11} \mathrm{Sn}$ is $2.3 \mathrm{meV}$, whereas in $\mathrm{Tl}_{0.8} \mathrm{Co}_{3} \mathrm{FeSb}_{12}$ the observed width is resolution limited. Hence, $w_{\text {nat }}$ is estimated to be less than $0.9 \mathrm{meV}$ if the maximum error on the observed linewidth, $w_{\text {obs }}$, is used. The well-defined character of the local mode in $\mathrm{Tl}_{0.8} \mathrm{Co}_{3} \mathrm{FeSb}_{12}$ is an indication that the motion of the thallium atom is more harmonic than rattling. If this harmonic description is correct, the reduction in thermal conductivity observed for the filled skutterudites should be attributed to the independent incoherent and localized motion of the filling atoms, rather than to any anharmonicity in the motion of the filling atom. In other words, the reduced thermal conductivity is caused by local Einstein oscillators that are randomly distributed in phase, a random distribution that breaks the translational symmetry and leads to Rayleigh scattering of the phonons.

It should be noted that several features of the DOS models calculated by Feldman and Singh [7] are observed in the experimental DOS of $\mathrm{CoSb}_{3}$ in spite of the instrumental broadening. These features include a parabolic dependence up to $80 \mathrm{~cm}^{-1}$, i.e., $10 \mathrm{meV}$, a gap around $110 \mathrm{~cm}^{-1}$, i.e., $13 \mathrm{meV}$, and a gap at $210 \mathrm{~cm}^{-1}$, i.e., $23 \mathrm{meV}$. Our instrumental resolution at these energy transfers is 4,5 , and $10 \mathrm{meV}$, respectively. Two broad peaks were observed [3] at $\sim 7$ and $14 \mathrm{meV}$ in the inelastic neutron scattering difference between $\mathrm{LaFe}_{4} \mathrm{Sb}_{12}$ and $\mathrm{CeFe}_{4} \mathrm{Sb}_{12}$ which have been discussed in a recent lattice dynamics study [8]. In contrast, our inelastic neutron scattering measurements on the thallium filled skutterudites reveal only a single well-defined excitation peak at an energy of $\sim 5 \mathrm{meV}$. The difference between 5 and $7 \mathrm{meV}$ energies is, however, not surprising given the higher atomic mass of $\mathrm{Tl}$ as compared to La.

In conclusion, the heat capacity measurements on $\mathrm{Tl}_{0.8} \mathrm{Co}_{4} \mathrm{Sb}_{11} \mathrm{Sn}$ indicate an Einstein vibrating behavior for the thallium atoms. The inelastic neutron scattering measurements on $\mathrm{Tl}_{0.8} \mathrm{Co}_{4} \mathrm{Sb}_{11} \mathrm{Sn}, \mathrm{Tl}_{0.5} \mathrm{Co}_{3.5} \mathrm{Fe}_{0.5} \mathrm{Sb}_{12}$, and $\mathrm{Tl}_{0.8} \mathrm{Co}_{3} \mathrm{FeSb}_{12}$ show a localized vibrational mode, which confirms this Einstein vibrational behavior. Further, the $56 \pm 2 \mathrm{~K}$ temperature of the Einstein vibrational mode obtained from the heat capacity measurements is in excellent agreement with the $5 \mathrm{meV}$ energy or $57 \pm 2 \mathrm{~K}$ values obtained from the neutron scattering measurements.

The inelastic neutron scattering experiments were supported by EU Grant No. UDS-01-012. Oak Ridge National Laboratory is managed by UT-Battelle, LLC, for the U.S. Department of Energy under Contract No. DE-AC0500OR22725.

*To whom correspondence should be addressed. Email address: fgrandjean@ulg.ac.be

[1] G. A. Slack, in Thermoelectric Materials in CRC Handbook of Thermoelectrics, edited by D. M. Rowe (Chemical Rubber Co., Boca Raton, FL, 1995), Chap. 34.

[2] B.C. Sales, D. Mandrus, and R. K. Williams, Science 272, 1325 (1996).

[3] V. Keppens, D. Mandrus, B.C. Sales, B.C. Chakoumakos, P. Day, R. Coldea, M. B. Maple, D. A. Gajewski, E. J. Freeman, and S. Bennington, Nature (London) 395, 876 (1998). 
[4] B. C. Sales, B. C. Chakoumakos, and D. Mandrus, Phys. Rev. B 61, 2475 (2000).

[5] G. J. Long, B. Mahieu, B. C. Sales, R. P. Hermann, and F. Grandjean, J. Appl. Phys. 92, 7236 (2002).

[6] W. Schweika and P. Böni, Physica (Amsterdam) 297B, 155 (2001).
[7] J. L. Feldman and D. J. Singh, Phys. Rev. B 53, 6273 (1996).

[8] D. J. Singh, I. I. Mazin, J. L. Feldman, and M. Fornari, Mat. Res. Symp. Proc. 545, 3 (1999); J. L. Feldman, D. J. Singh, I. I. Mazin, D. Mandrus, and B.C. Sales, Phys. Rev. B 61, R9209 (2000). 